\title{
EB Mastering Process for SIL Readout/Recording System
}

\author{
Motohiro Furuki, Minoru Takeda, Masanobu Yamamoto, \\ Kimihiro Saito, Masataka Shinoda, Toshihiro Fujiki, Yuichi Aki* \\ Storage Technologies Department, Optical System Development Div., HENC, Sony Corporation \\ *Manufacturing Eng. Dev. Ctr., MSNC, Sony Corporation \\ 6-7-35 Kitashinagawa, Shinagawa-ku, Tokyo 141-0001 Japan \\ Mitsuru Koizumi, Toshiaki Miyokawa, Masao Muto \\ Advanced Technology Div., JEOL Ltd. \\ 3-1-2, Musashino, Akishima-shi, Tokyo 196-8558 Japan
}

\begin{abstract}
We improved the electron beam recorder with a differential pumping head for higher density discs and mass production. The beam diameters were improved by changing the aperture size of the objective lens, and beam stability was also improved by adding a sound proof case. As for the performance of the improved electron beam recorder, we have demonstrated the capability of $70 \mathrm{~Gb} / \mathrm{in}^{2}$ density recording by an electron beam mastering process and readout by a solid-immersion lens with a blue LD of $405 \mathrm{~nm}$ wavelength and 2.05NA. In addition, we improved the pit width uniformity by introducing an appropriate write strategy that was simulated by a Monte Carlo simulation to the recording pulses. Thus we were able to fabricate from a $104 \mathrm{~Gb} / \mathrm{in}^{2}$ to $150 \mathrm{~Gb} / \mathrm{in}^{2}$ ROM disc by using the write strategy. For the solid-immersion lens recording system with the blue LD and 1.84NA, we obtained the clear eye patterns of $73 \mathrm{~Gb} / \mathrm{in}^{2}$ in which the groove track pitch is $160 \mathrm{~nm}$ by utilizing a phase change recording media and a polycarbonate substrate.
\end{abstract}

Keywords: electron beam mastering, near-field optical pick-up, reactive ion etching, write strategy

\section{Introduction}

A higher density disk has been recently developed in the optical disk field for the next generation after Blu-ray Disc. The combination of electron beam (EB) mastering and a solid-immersion lens (SIL) readout/recording system is one of the most promising approaches to achieve more than $100 \mathrm{~Gb} / \mathrm{in}^{2}$ density disks. We have developed an electron beam recorder \#1 (EBR) with a novel differential pumping head, which can be very useful for mass production of a Blu-ray Disc ROM (BD-ROM) whose capacity is from $23.3 \mathrm{~GB} /$ layer to $27 \mathrm{~GB} /$ layer, and even higher densities because it is very compact and enables us to fabricate the BD-ROM at a higher mastering velocity compared to a conventional EBR. ${ }^{1)}$ To try a higher density, we have further fabricated $36 \mathrm{~Gb} / \mathrm{in}^{2}$ (50GB capacity/layer) and optimized Si etching processes to form suitable pit pattern shapes. ${ }^{2)}$ A clear eye pattern was obtained by the use of a near-field optical pick-up with a $405 \mathrm{~nm}$ wavelength $\mathrm{LD}$ and the SIL type objective of $1.84 \mathrm{NA}$, and a signal jitter value of
9.6\% with a limit equalizer was achieved. ${ }^{3)}$ In addition, we applied the same schematic etching processes to $70 \mathrm{~Gb} /$ in $^{2}$ density (100GB capacity/layer) and demonstrated the capability of playing back an acceptable RF signal with a near field optical pick-up with a $405 \mathrm{~nm}$ LD and a SIL type objective of 2.05NA. ${ }^{2-4)}$

Although EB lithography tools are capable of realizing a higher density disk, the backscattered electrons cause a proximity effect, where the dose that a pattern feature receives is affected by electron scattering from other features nearby. In addition, it is hard to control various pit mark sizes for more than $100 \mathrm{~Gb} / \mathrm{in}^{2}$ density with a chemically amplified resist that has a non-linear sensitivity.

In this paper, we describe our concept EBR with a differential pumping head and improved points for fabricating higher density discs and mass production. We report the suitable pit shape for the SIL readout system by applying the additional reactive ion etching (RIE) process to more than $36 \mathrm{~Gb} / \mathrm{in}^{2}$. We also show the recent progress of the EB mastering for from $100 \mathrm{~Gb} / \mathrm{in}^{2}$ to $150 \mathrm{~Gb} / \mathrm{in}^{2} \mathrm{ROM}$ density discs with an appropriate write strategy. Finally, we report the results of a SIL recording with a phase change media and a polycarbonate substrate.

\section{Experimental Conditions}

\subsection{Electron Beam Recorder (Nano Code Cutter ${ }^{\mathrm{TM}}$ )}

We newly developed the EBR\#2 for higher density mastering and mass production, which is called Nano Code Cutter, as shown in Fig. 1.

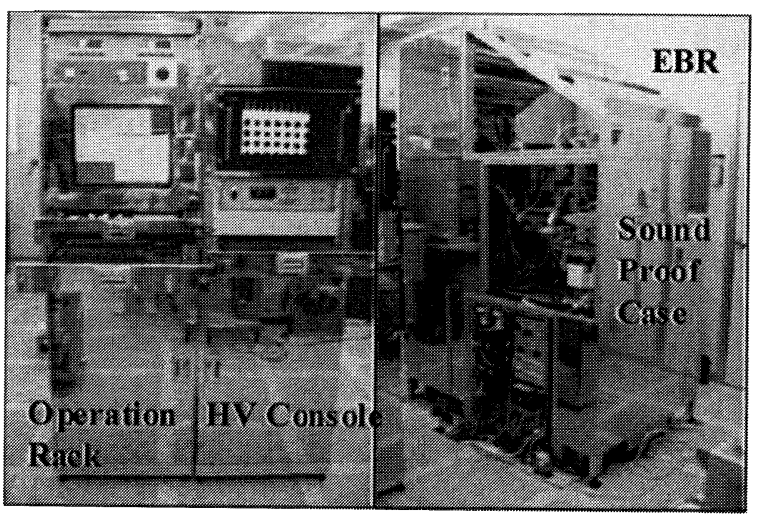

Fig. 1 Nano Code Cutter ${ }^{\mathrm{TM}}$ (electron beam recorder) 
The Nano Code Cutter is comprised of an operation rack, high voltage console and the EBR. The size of the compact operation rack and high voltage console is about $1.14 \mathrm{~m}(\mathrm{~W}) \times 0.85 \mathrm{~m}(\mathrm{D}) \times 1.85 \mathrm{~m}(\mathrm{H})$, and that of the EBR is $1.1 \mathrm{~m}(\mathrm{~W}) \times 1.6 \mathrm{~m}(\mathrm{D}) \times 2.2 \mathrm{~m}(\mathrm{H})$, which can be very useful for mass production optical disk manufactures. We improved beam stability against the electron charge up and mechanical vibration caused by acoustic noise, by adding a sound proof case in order to reduce the sound pressure.

Fig. 2 shows the schematic of the electron beam column and the differential pumping head (DPH) connected to its bottom portion.

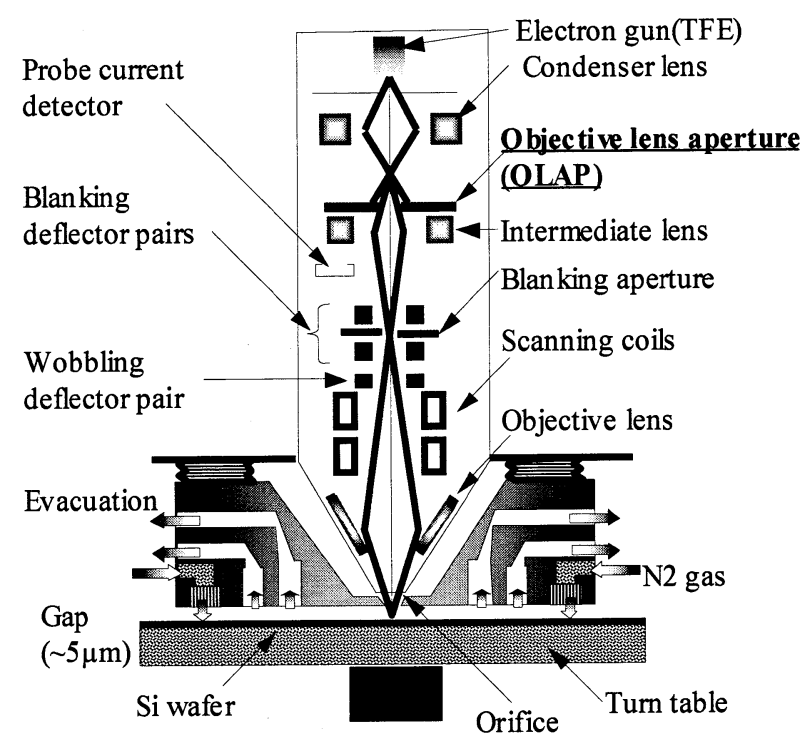

Fig. 2 optical schematic of the electron column and the structure of the diffe rential pump ing head

The electron gun is of a $\mathrm{ZrO} / \mathrm{W}$ thermal-field emission type and the maximum accclcration voltage is $15 \mathrm{kV}$. The low acceleration voltage makes the resist sensitivity very high and is safe for the operator as it prevents X-ray scattering. The electron beam is focused on the blanking aperture by the intermediate lens, and is deflected to scan across it. The beam blanker for turning the beam on and off consists of two pairs of blanking deflectors driven at the same phase and a beam-blanking aperture. The deflection angle required for the beam blanking is so small that the rise and fall time of the beam can be shortened to approximately 1 ns very effectively. As a result, a much higher frequency of more than 50 $\mathrm{MHz}$ was achieved using this beam blanker, compared to the simple conjugate blanker. One of the blanking pairs is used to dynamically correct the spherical aberration caused by beam motion during the blanking and unblanking processes. In addition, wobbling deflectors were also introduced under the beam blanker to fabricate various wobbling patterns in the case of different optical disk formats. Then the objective lens is conically shaped to fit the surrounding differential pumping head at the bottom portion of the column. A Faraday cup is mounted below the intermediate lens to measure the beam current in order to ensure the correct dose for resist exposure.

The differential pumping head produces a local high-vacuum area for the electron beam trajectory by balancing nitrogen gas flow into and out of the head. In order to float the DPH from the substrate, compressed nitrogen gas is injected at $5 \times 10^{5} \mathrm{~Pa}$ into the head from the outer ring that is made of porous ceramic carbon. The head floats at about $5 \mu \mathrm{m}$ constantly from the resist-coated Si substrate by the air-suspension method reported previously. Then inner areas are pumped down to about $10^{2}$ and $10^{\circ} \mathrm{Pa}$ from the two inner concave rings by a dry pump at a pumping speed of $2501 / \mathrm{min}$, and the center area with a $0.8 \mathrm{~mm}$ orifice for the electron beam trajectory is pumped down to $10^{-3} \mathrm{~Pa}$ by a turbo molecular pump (TMP) at $300 \mathrm{l} / \mathrm{s}$. Regulation of the nitrogen pressure controls the gap distance between the DPH and the resist-coated Si wafer. A large pressure gradient is formed in this narrow gap and a vacuum condition of less than $10^{-3} \mathrm{~Pa}$ is achieved at the central region where the electron beam propagates from the aperture to the resist surface. As it takes only a few minutes to evacuate the narrow gap region to reach the required vacuum quality, the total exchange time of Si substrates is approximately 2 min.

To fabricate more than $100 \mathrm{~Gb} / \mathrm{in}^{2}$, the beam diameter was also improved by changing the aperture size of the objective lens (OLAP), as shown in Fig. 3. The beam diameters at $15 \mathrm{kV}$ acceleration voltage were measured by the knife-edge method. Fig. 3 shows the beam diameter dependence on the beam current compared to simulations when the aperture size of the objective lens is $50 \mu \mathrm{m}$ $(E B R \# 1)$ and $80 \mu \mathrm{m}(E B R \# 2)$, respectively. We obtained a $35 \mathrm{~nm}$ beam diameter (FWHM) at 20nA probe current with an $80 \mu \mathrm{m}$ OLAP, which has improved approximately $20 \%$ over the prototype EBR\#1. This performance is also appropriate for fabricating $100 \mathrm{~Gb} / \mathrm{in}^{2}$ density discs.

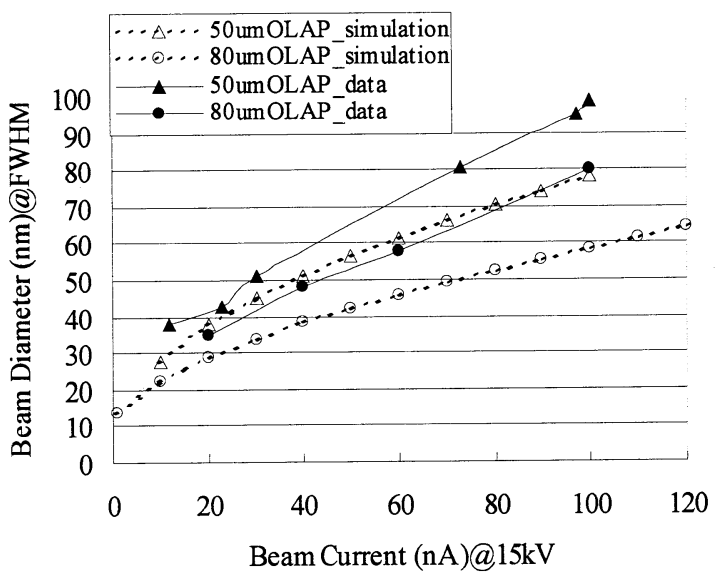

Fig. 3 Beam diameter vs. beam current

\subsection{Mastering Process}

First of all, Si wafers covered with a chemically amplified EB resist are prepared for recording pit patterns. After the resists are exposed by an EB and a 
post-exposure baking (PEB) process are applied to the substrate, pit patterns are formed using an alkaline developer. Then, for the more than $36 \mathrm{~Gb} / \mathrm{in}^{2}$ density, the resist pattern on the $\mathrm{Si}$ wafer was transferred into the $\mathrm{Si}$ substrate by a RIE method.

After removing the resist and cleaning the surface of $\mathrm{Si}$, the pit pattern shape was observed by an atomic force microscope (AFM) and the etched Si disc without cover layer was directly read out by a near field optical pick-up with a $405 \mathrm{~nm}$ wavelength LD and a SIL type objective. When signal modulation or asymmetry of the actual eye pattern was not sufficient, additional Si etching process steps by oxygen or $\mathrm{CF}_{4}$ gas was performed. Following this the readout signal was evaluated repeatedly, so the optimum pit shape and RIE process for maximizing signal quality can be pursued accordingly. ${ }^{2)}$

Fig. 4 shows the schematic diagram of the near-field readout optical system. The air gap between the Si disc surface and the flat surface of the SIL was controlled by utilizing the total reflection on the surface of the SIL, because the polarization changes occur on surfaces of the SIL. ${ }^{6}$ The incident polarization on the disc is circular. Owing to the combination of the QWP and the PBS, basically all the light reflected from the disc reaches the photo detector for the RF signal. However, the light through the PBS is also detected by the same photo detector and is used as the air gap control signal. The air gap is controlled to approximately $25 \mathrm{~nm}$. The tracking servo signal is obtained with a push-pull tracking method.

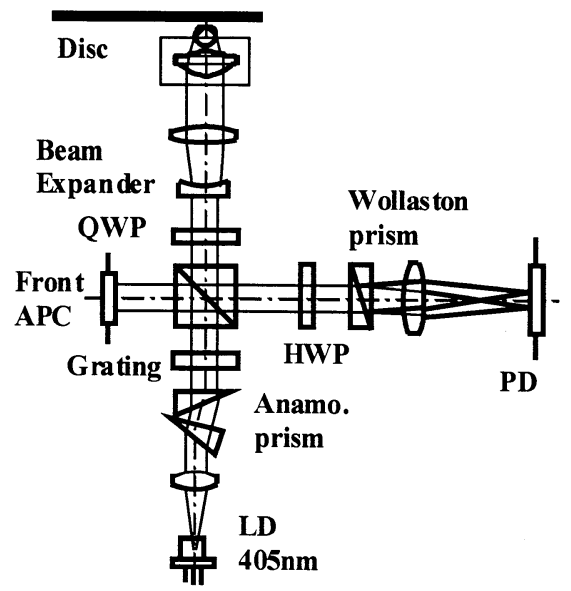

Fig. 4 Diagram of the near-field optical system

\subsection{Monte Carlo Simulation}

We further introduced some write strategy to the recording pulse to increase a margin of the process and tried to fabricate $104 \mathrm{~Gb} / \mathrm{in}^{2}$ density. To optimize the write strategy for fabricating more than $100 \mathrm{~Gb} / \mathrm{in}^{2}$ with $\mathrm{EFM}+$ modulation codes, the Monte Carlo simulation was used with a VS-M/EB Ver. 6 application software (Fuji Research Institute Corporation). We summarize the analysis conditions of the Monte Carlo simulation in Table 1. The distribution of energy deposition in the positive chemically amplified (CA) resist was simulated for exposure on a Si substrate at a beam energy of $15 \mathrm{keV}$ with a diameter of $30 \mathrm{~nm}$ (FWHM) and beam current of
$8 \mathrm{nA}$. A resist thickness of $50 \mathrm{~nm}$ is assumed and a total of 500, 000 electron trajectories are calculated in a range up to $13 \mu \mathrm{m}$.

Table 1 Analys is condition of the Monte Carlo simulation

\begin{tabular}{ll}
\hline $\begin{array}{l}\text { Incident beam conditions } \\
\text { beam energy }\end{array}$ & $15 \mathrm{keV}$ \\
beam diameter@FWHM & $30 \mathrm{~nm}$ \\
beam current & $8 \mathrm{nA}$ \\
\hline $\begin{array}{l}\text { Structure conditions } \\
\text { substrate }\end{array}$ & $\mathrm{Si}$ \\
resist type & positive CA resist \\
resist thickness & $50 \mathrm{~nm}$ \\
\hline Scanning geometry & \\
track pitch & $130 \mathrm{~nm}$ \\
min. pit & $71.4 \mathrm{~nm}$ \\
modulation code & $\mathrm{EFM}+$ \\
scanning area & $13000 \mathrm{~nm}^{\square}$ \\
number of electron trajectory & 500,000 \\
\hline
\end{tabular}

\section{Results and discussion}

\subsection{ROM disc}

To readout higher density discs with a near-field optical system (NA1.84), 36Gb/in ${ }^{2}$ density (50GB/layer) pit patterns using a 17PP modulation scheme were recorded and transferred to the $\mathrm{Si}$ substrate by a RIE etching process, in which the track pitch is $226 \mathrm{~nm}$ and the minimum pit length is $105 \mathrm{~nm}^{2}$.) Although the pit pattern shape was observed to be sufficient and resolved clearly by AFM, the asymmetry of the eye pattern and the $2 \mathrm{~T} / 8 \mathrm{~T}$ modulation ratios were insufficient. It is likely that the large angle of incidence of the light ray from the near-field objective lens needs a more widely opened pit shape to achieve the appropriate signal asymmetry and modulation amplitude. The reason for this is that the quality of the RF signal was improved gradually by performing the additional etching processes with oxygen and $\mathrm{CF}_{4}$ gas to the $\mathrm{Si}$ substrate, as shown in Fig. 5. As the pit edge angle changed from 80 to 50 degrees with the additional etching processes, the normalized amplitude of $2 \mathrm{~T}$ and $8 \mathrm{~T}$ marks increased to $60 \%$ and $50 \%$, respectively. As a result of that, a signal jitter value of $9.6 \%$ with a limit cqualizer was achieved from the additional etched Si disc. This experimental result is strongly supported by that of simulation as well.

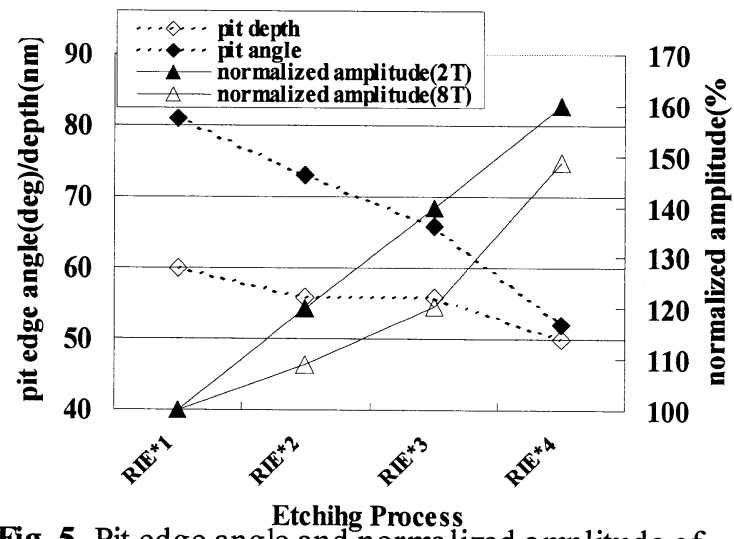

Fig. 5 Pit edge angle and normalized a mplitude of the RF signal for various Si RIE process steps 
We also applied the same scheme of the etching process to the $70 \mathrm{~Gb} / \mathrm{in}^{2}$ (100GB/layer) with an EFM plus modulation scheme and obtained an acceptable RF signal with a SIL type of objective lens $2.05 \mathrm{NA}$ and $405 \mathrm{~nm}$ LD, as shown in Fig. 6. Fig. 6(a) shows an SEM image of $70 \mathrm{~Gb} / \mathrm{in} 2$ whose track pitch is $160 \mathrm{~nm}$ and shortest pit is $87 \mathrm{~nm}$. We obtained reasonable $3 \mathrm{~T} / 11 \mathrm{~T}$ modulation ratio that is approximately $34 \%$ compared to a theoretical curve that is calculated using the scalar diffraction theory. ${ }^{6}$ However, we see from Fig. 6(b) that the RF signal is noisy. It is likely that cross talk and inter-symbol interference might be the predominantly influential elements to make the RF signal slightly noisy because the pit shapes are widely opened by additional etching processes.

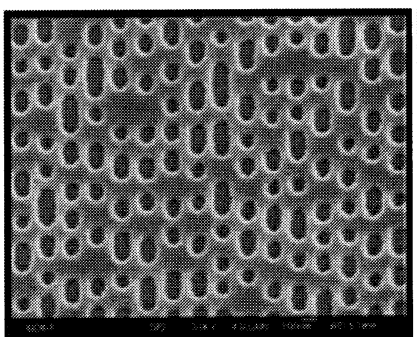

(a)

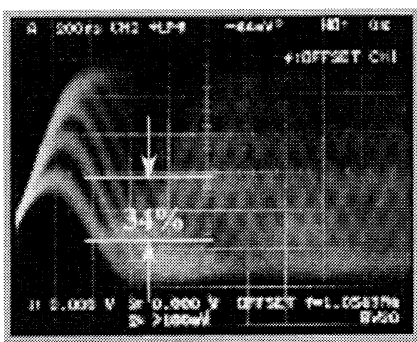

(b)
Fig. 6 (a) SEM image of $70 \mathrm{~Gb} / \mathrm{in}^{2}$ (100GB/layer) track pitch $=160 \mathrm{~nm}$, min.pit length $=87 \mathrm{~nm}, \mathrm{EFM}+$

(b) Eye pattern of RF signal corresponding to $70 \mathrm{~Gb} / \mathrm{in}^{2}$ using a near-field optical pick up 2.05NA

We further tried to fabricate a $104 \mathrm{~Gb} / \mathrm{in}^{2}$ (150GB capacity/layer) density disc with an EBR\#2 whose beam spot size is smaller than that of the proto type EBR\#1. EFM plus modulation code patterns were recorded with track pitches of $130 \mathrm{~nm}$ and minimum pits size of $71 \mathrm{~nm}$.

Under typical experimental recording conditions, the beam current is approximately $7 \mathrm{nA}$, the acceleration voltage is $15 \mathrm{kV}$, and the recording velocity is $1.19 \mathrm{~m} / \mathrm{s}$. The beam diameter is assumed to be less than $30 \mathrm{~nm}$ (FWHM), as shown in Fig. 3. We confirmed that it is possible to fabricate a $104 \mathrm{~Gb} / \mathrm{in}^{2}$ density disc by using the EBR\#2 and optimizing the mastering process conditions including EB dose, pre-baking, post exposure baking (PEB) and post-baking conditions.

However, a process margin is becoming smaller because of the following reasons. First of all, it is caused by a characteristic of a chemically amplified resist. There is a significant difference in the pit width between $3 \mathrm{~T}$ and $11 \mathrm{~T}$ marks with EFM plus modulation, approximately $40 \mathrm{~nm}$. This results from principle reactions of the chemically amplified resist, which is due to the difference in the amount of the initial concentration of the acid generator between shorter and longer pits. In addition, it is caused by the electron-solid interactions. Using a thinner resist is also necessary to fabricate more than $100 \mathrm{~Gb} / \mathrm{in}^{2}$ because of the broader beam profile at the bottom of the resist than at the top, especially in the case of a low acceleration voltage. The increase in effective beam diameter in nanometers due to the forward scattering is given empirically by the formula $\sigma_{\mathrm{f}}=0.9 \times(\mathrm{T} / \mathrm{V}){ }^{1.5}$, where $\mathrm{T}$ is the resist thickness in nanometers and $\mathrm{V}$ is the beam voltage in kilovolts. As an example, the forward scattering diameter of $100 \mathrm{~nm}$ resist thickness is approximately three times as much as that of $50 \mathrm{~nm}$ thickness at $15 \mathrm{kV}$.

Finally, the size of the pit pattern after applying RIE with $\mathrm{CF}_{4}$ gas to a Si substrate diminishes approximately $25 \mathrm{~nm}$ uniformly for each pit length. As a result, the ratio of the pit width between $3 \mathrm{~T}$ and $11 \mathrm{~T}$ marks in the case of the resist pattern decreases from $70 \%$ to $58 \%$ in comparison to the Si substrate by performing the RIE etching. This fact implies the small amplitude of 3T mark and insufficient asymmetry are obtained from the readout signal. Although the additional RIE etching process steps to the $70 \mathrm{~Gb} / \mathrm{in}^{2}$ density disc were effective in maximizing the resolution and adjusting the asymmetry of the RF signal, the same scheme to the $104 \mathrm{~Gb} / \mathrm{in}^{2}$ made some minimum pits disappear because of the shallow etched depth. In order to solve the problems, it is necessary to fabricate a uniform width for each pit size by the EB lithography. To take an example, it is reported that applying the write strategy for longer pits is useful for decreasing the cross talk from the readout signal with BD-ROM. ${ }^{\text {s) }}$

In this experiment, about $104 \mathrm{~Gb} / \mathrm{in}^{2}$ density, we tried to optimize the write strategy with the Monte Carlo simulation before the exposure experiments because there are quite a number of write strategies. In the case of the EB lithography, it is significant to consider the proximity effect caused by the forward and back scattering, the characteristics of the CA resist as mentioned above and the change in the pattern size after applying RIE to the Si.

To take but one example, the distribution of the energy deposition in the resist and the profile after development are shown in Fig. 7. Fig. 7(a) shows the normal case and 7(b) shows the case with the write strategy within a $0.6 \mu \mathrm{m}$ square. These distributions of the energy deposition are shown at the height of $25 \mathrm{~nm}$ from the Si surface and the acid diffusion coefficient of the chemically amplified is assumed to be $21.2 \mathrm{~nm}$. In the case of using the write strategy, each recording pulse was divided up into some pulses. The energy deposition for longer pits with the write strategy is obviously narrower than that with the normal recording pulse.

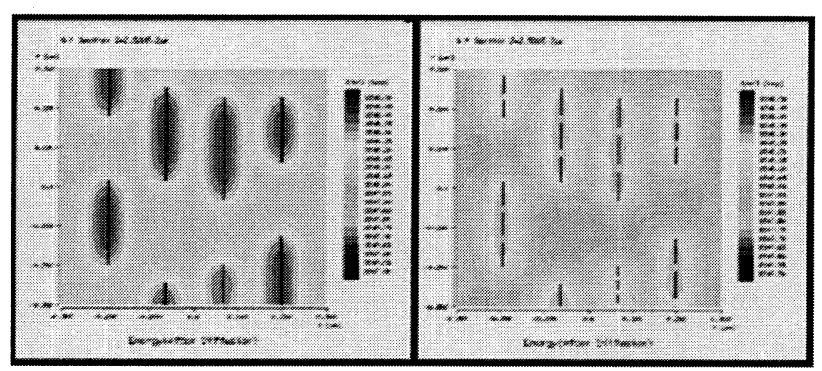

(a)

(b)

Fig. 7 Distribution of energy deposition in the resist:

(a) normal case, (b) write strategy case 
About the profile after the development, Mack Model was used to estimate the development speed ${ }^{6}$, and the pit length and width for each mark were calculated. Based on these results, the appropriate write strategy for realizing a uniform pit width at $104 \mathrm{~Gb} / \mathrm{in}^{2}$ was simulated and applied it to the EB mastering.

We fabricated the $104 \mathrm{~Gb} / \mathrm{in}^{2}$ (150GB/layer) with the same EB current per unit time and the SEM images of the etched $\mathrm{Si}$ disc are shown in Fig. 8. In the case of the normal recording pulse, the difference of the pit width between $3 \mathrm{~T}$ and $11 \mathrm{~T}$ marks is over $30 \mathrm{~nm}$ and some pits are occasionally merging and deformed, as shown in Fig. 8(a). On the other hand, that of the write strategy case is almost uniform and improved to approximately $10 \mathrm{~nm}$ between $3 \mathrm{~T}$ and $11 \mathrm{~T}$ marks, as shown in Fig. 8(b). We successfully improved pit width uniformity by introducing an appropriate write strategy that results from the Monte Carlo simulation. An acceptable RF signal with the high resolution and appropriate asymmetry will be obtained from the improved $\mathrm{Si}$ disc, using the near-field optical pick-up readout system.

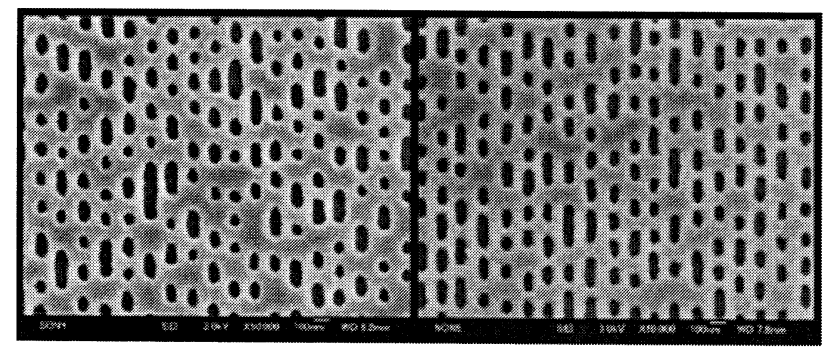

(a)

(b)

Fig. 8 SEM images of $104 \mathrm{~Gb} / \mathrm{in}^{2}$ (150GB/layer)

$\mathrm{EFM}+: \mathrm{tp}=130 \mathrm{~nm}, \mathrm{~min}$. pit $=71 \mathrm{~nm}$, pit depth=60n m

(a) normal case, (b) a write strategy case

We finally tried to fabricate a $150 \mathrm{~Gb} / \mathrm{in}^{2}$ (220GB/layer) by applying a write strategy to EFM+ modulation codes whose track pitch is $108 \mathrm{~nm}$ and minimum pit length is $58.6 \mathrm{~nm}$, as shown in Fig. 9. We confirmed that it is possible to fabricate a $150 \mathrm{~Gb} / \mathrm{in}^{2} \mathrm{ROM}$ disc by optimizing the write strategy to the recording pulses.

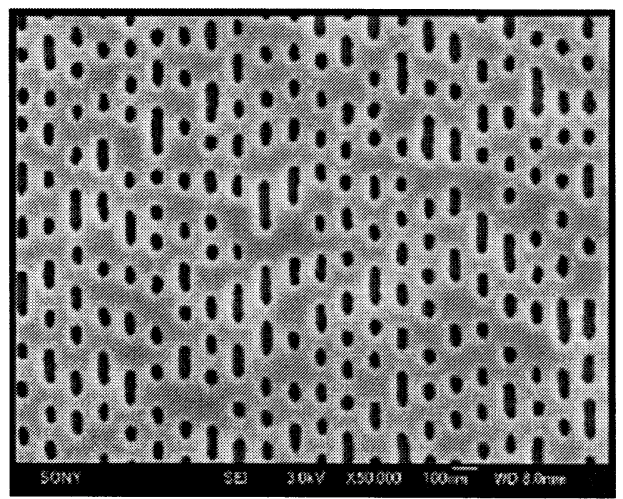

Fig. 9 SEM image of $150 \mathrm{~Gb} / \mathrm{in}^{2}$ (220GB/layer) $\mathrm{EFM}+$, track pitch $=108 \mathrm{n} \mathrm{m}$, min.p it length $=58.6 \mathrm{~nm}$

\subsection{Recordable disk}

Furthermore, we have investigated that a near-field recording system using a phase change media and molded polycarbonate substrate. $^{8)}$ Fig. 10(a) shows the etched groove patterns whose track pitch is $160 \mathrm{~nm}$ and a groove depth is $20 \mathrm{~nm}$ and groove duty is approximately $35 \%$. Then a Ni stamper was duplicated with the etched $\mathrm{Si}$ wafer, and polycarbonate substrates were replicated by molding on the stamper. Fig. 10(b) shows a structure of a phase change recording media. Any hard coat and lubricant film were not used for protecting the disc surface. It is necessary that a refractive index of the first layer is larger than NA of the SIL. Finally, as an example of the near-filed optical recording, we show the RF signal of the $73 \mathrm{~Gb} / \mathrm{in}^{2}$ density whose bit length is $55 \mathrm{~nm}$ using a near-field recording system with a $405 \mathrm{~nm}$ wavelength LD and the SIL type objective of $1.84 \mathrm{NA}$, as shown in Fig. 11. 17PP modulation code patterns were recorded on the on-groove area of $160 \mathrm{~nm}$ track pitches. It should be noted that the adjacent tracks were not recorded in this experiment. As a result, we successfully obtained the clear eye pattern without a limit equalizer from this recording media and reasonable $2 \mathrm{~T} / 8 \mathrm{~T}$ modulation ratio that is approximately $10 \%$.

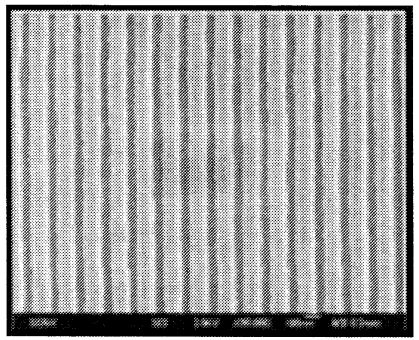

(a)

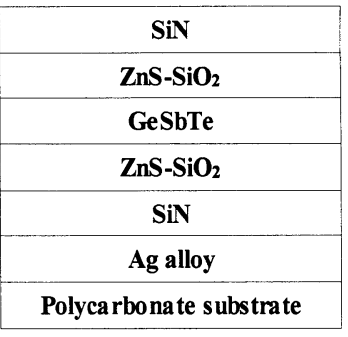

(b)
Fig. 10 (a)SEM images of groove pattern $\mathrm{tp}=160 \mathrm{~nm}$, groove depth $=20 \mathrm{~nm}$. Groove duty $=35 \%$

(b) a structure of a phase change media

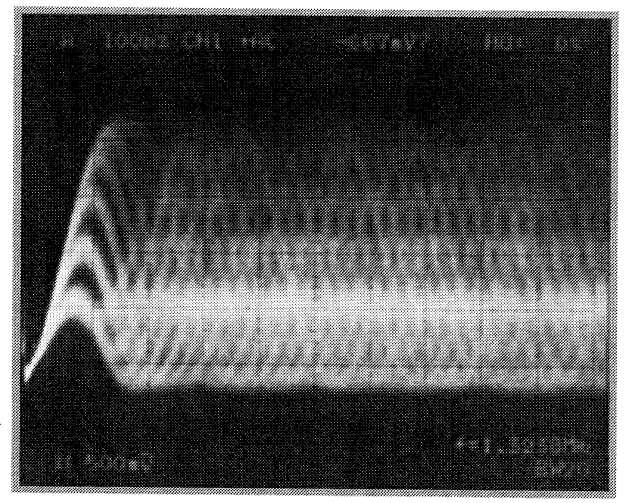

Fig. 11 Eye pattern of RF signal corresponding to $73 \mathrm{~Gb} /$ in $^{2}$ using a near-field optical pick up of $1.84 \mathrm{NA}$ bit length $=55 \mathrm{n} \mathrm{m}$

In Fig. 12, the $2 \mathrm{~T} / 8 \mathrm{~T}$ and $3 \mathrm{~T} / 11 \mathrm{~T}$ modulation ratios of these disks are plotted with several values we mentioned 
above. The curves for 1.84NA and 2.05NA in this figure were calculated using the diffraction theory. ${ }^{6}$ These experimental results are in good agreement with the theoretical ones. We confirmed that these optical pick up realized effective NA of 1.84 and 2.05, respectively and our SIL system was able to read out/write higher density disks.

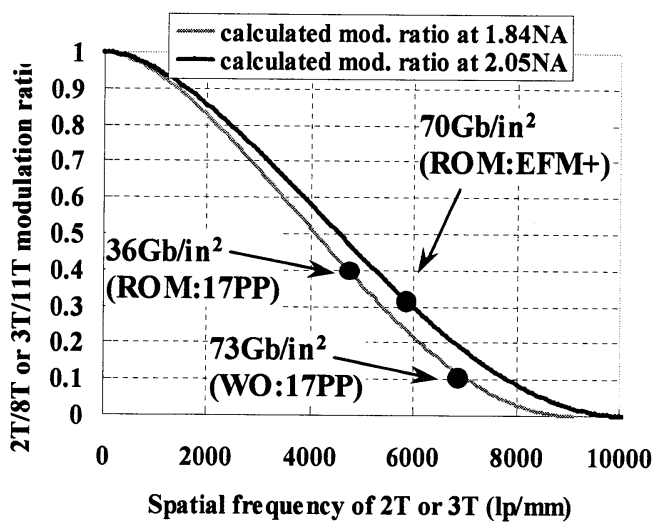

Fig. 12 Calculated and measured $2 \mathrm{~T} / 8 \mathrm{~T}$ and $3 \mathrm{~T} / 11 \mathrm{~T}$ modulation ratios

\section{Conclusion}

We improved the EBR with a differential pumping head, which is called Nano Code Cutter ${ }^{\mathrm{TM}}$ for higher density discs and mass production of a Blu-ray Disc. The beam diameters were improved by changing the aperture size of the objective lens. In addition, beam stability against the electron charge up and mechanical vibration caused by acoustic noise were improved by adding a sound proof case. We reported the suitable pit shape for the SIL readout system by applying the additional RIE etching processes for from $36 \mathrm{~Gb} / \mathrm{in}^{2}$ (50GB/layer) to $70 \mathrm{~Gb} / \mathrm{in}^{2}$ (100GB/layer). Furthermore, we showed that a $104 \mathrm{~Gb} / \mathrm{in}^{2} \quad(150 \mathrm{~GB} /$ layer) and $150 \mathrm{~Gb} / \mathrm{in}^{2}(220 \mathrm{~GB} / \mathrm{layer})$ density disc with the EFM plus modulation codes can be fabricated by a Nano Code Cutter ${ }^{\mathrm{TM}}$, and we succeeded in fabricating almost uniform pit width for each pit length by introducing an appropriate write strategy that was simulated by the Monte Carlo simulation and in increasing the process margin. We finally demonstrated the recordable near-field optical disk whose density is $73 \mathrm{~Gb} / \mathrm{in}^{2}$ and a groove track pitch is $160 \mathrm{~nm}$. A clear eye pattern was obtained from a phase change media on a polycarbonate substrate by the use of a blue LD and 1.84NA SIL system. We confirmed that the combination of EB mastering and a SIL readout/write system is one of the most promising approaches to achieve higher density optical disks.

Acknowledgements The authors wish to thank Fuji Research Institute Corporation for supporting the electron beam analysis. The authors also wish to thank their colleagues, N. Mukai, A. Suzuki, Y. Miura of the Manufacturing Engineering Development Ctr., MSNC for help in developing the Nano Code Cutter ${ }^{\mathrm{TM}}$, and $\mathrm{M}$. Nakakura of MEITEC Corp. for the disc fabrication support.

\section{References}

1) M. Furuki, M. Takeda, M. Yamamoto, Y. Aki, H. Kawase, M. Koizumi, S. Takashima, T. Miyokawa, and N. Date: Jpn. J. Appl. Phys., 42, 759 (2003).

2) M. Furuki, M. Takeda, M. Yamamoto, M. Shinoda, K. Saito, Y. Aki, H. Kawase, M. Koizumi, T. Miyokawa and M. Muto: Technical Digest of Optical Data Storage Topical Meeting 2003, p. 62 (2003).

3) M. Takeda, M.Furuki, M. Yamamoto, M. Shinoda, K. Saito, Y. Aki, H. Kawase, M. Koizumi, T. Miyokawa and M. Muto: Proc. of SPIE, Vol. 5069, p. 209 (2003).

4) M. Shinoda, K. Saito, T. Ishimoto, T. Kondo, A. Nakaoki, M. Furuki, M. Takeda and M. Yamamoto: Technical Digest of Optical Data Storage Topical Meeting 2003, p. 120 (2003).

5) M. Tsukuda, E. Ito, Y. Kawaguchi, M. Tomiyama, S. Abe, S. Furukawa and E. Ohno: Jpn. J. Appl. Phys., 42, 1076 (2003).

6) K. Saito, T. Ishimoto, T. Kondo, A. Nakaoki, S. Masuhara, M. Furuki and M. Yamamoto: Jpn., J. Appl. Phys., 41, 1898 (2002).

7) C.A. Mack: Proc. SPIE 3236, p. 25 (1997).

8) M. Shinoda, K. Saito, T. Ishimoto, T. Kondo, A. Nakaoki, M. Furuki, M. Takeda, Y. Akiyama, T. Shimouma and M. Yamamoto: Proc. of SPIE, Vol. 5380, (2004) in press. 